\title{
Two transitions of thermosolutal natural convection in the presence of an external magnetic field
}

\author{
Badia Ghernaout $^{1}$, Djamel Ghernaout ${ }^{2}$, Said Bouabdallah ${ }^{1 *}$, Aissa Atia ${ }^{1}$ \\ ${ }^{1}$ Laboratory of Mechanics (LME), Department of Mechanical Engineering, University of Laghouat, \\ PO Box 37G, Laghouat 03000, Algeria \\ ${ }^{2}$ Department of Chemical Engineering, College of Engineering, University of Ha'il, PO Box 2440, \\ Ha'il 81441, Saudi Arabia
}

Email: fibonsaid@gmail.com

\begin{abstract}
This paper presents a numerical study of two transitions (onset/oscillatory) of thermosolutal natural convection (TSNC) under the effect of an external magnetic field (MF) in binary mixture. For this, a square enclosure filled with a binary mixture has been considered and exposed to opposing solute and thermal gradients. An external and uniform MF is applied separately in two directions, i.e., vertical and horizontal. The results are given for varying buoyancy ratio $(N=0.75,1.0$ and 1.25), Prandtl number $(P r=0.71)$ and Schmidt number $(S c=3.5)$. The obtained results show a good agreement with the experimental data available in the literature. We show a strong effect of the buoyancy ratio on the flow field, the thermal and solute structure. The onset of TSNC and oscillatory TSNC flow are determined, and then the oscillatory flow occurs for a periodic time evolution where the phenomena change around in each period time.
\end{abstract}

Keywords: Thermosolutal Natural Convection (TSNC), Magnetic Field (MF), Oscillatory Flows, Onset Flow, Buoyancy Ratio.

\section{INTRODUCTION}

The effect of a magnetic field (MF) on the thermosolutal natural convection (TSNC) of a binary fluid has been less investigated in the open literature despite its large applications such as in solidification of metal alloys, nuclear fuel debris removal, geothermal reservoirs, etc. Among the experimental references on the thermosolutal convection, we may include experiences of Lee et al. [1], Kamotani et al. [2], and Kuehn and Han [3]. They used an electrochemical system comprising an anode and a cathode to force concentrations on the walls of the cavity. Lee et al. [1] examined the thermosolutal convection in a cavity filled with salt water. Depending on the value of the ratio of volume forces, they observed two types of convective motions: one with a single cell structure and the other with a multi-cell structure. In addition, the number of cells in the case of convection with opposing gradients is much greater than that observed in the case of convection cooperating with gradients. Kuehn and Han [3-4] have made experimental and numerical studies using an electrochemical technique with vertical electrodes maintained at different temperatures by performing photographs showing the evolution over time of multicellular structures in a cavity in the case of cooperating and opposing buoyancy forces. They also presented the characteristics of the multistage flow that include temperature profiles and concentration and characteristics of the heat and mass transfer. Chen et al. [5] found that, for finite and infinite layers, the linear stability analysis shows that: when the inclination of the cavity with respect to the gravity decreases from 0 to $90^{\circ}$, the threshold for stationary instability increases exponentially while the oscillatory instability threshold decreases exponentially. Zhou and Zebib [6] used a two-dimensional model of the solidification of pure metals in a horizontal rectangular cavity. They examined the influence of oscillatory transition of natural convection and predicted the parameters associated with this phenomenon. In particular, the influence of the solidification temperature on the critical Grashof number. Sezai and Mohamed [7] reported that the flow of the double diffusion, in cavities with opposing buoyancy forces, is strictly threedimensional for a range of the parameters: $(10<R a<2$ $\times 10^{5}$ ), buoyancy ratio: $-2<N<0$ and Lewis number: $0.1<$ $L e<150$. The same configuration was studied by Maatki et al. [8], but with the presence of the MF. They presented the structure of the three-dimensional flow, the distribution of temperature and concentration and the different characteristics of heat and mass transfer of the thermal and solutal dominated region.

Other studies related to the double diffusion problem in solar distiller can be found in literature such as Ghachem et al. [9] where numerical results of TSNC are presented in a three-dimensional. The flow is considered laminar and caused by the interaction of the thermal energy and the chemical species diffusions. Equations of concentration, energy and momentum are formulated using vector potential- 
vorticity formulations in its three-dimensional form, then solved by the finite volume method. The Rayleigh number is fixed at $R a=10^{5}$ and the effects of the buoyancy ratio are studied for opposed temperature and concentration gradients, with a particular interest to the three-dimensional aspects and entropy generation.

Bouabdallah and Bessaih [10] examined the effect of MF on $3 \mathrm{D}$ heat and fluid flow during phase change of a liquid metal. They found that the MF causes a significant control of the shape of the solid/liquid interface, and for high values of the Hartmann number $(H a=100)$, the convection is suppressed and the interface is regular. They have also showed that the MF causes a bidimensionalisation of the heat transfer along isothermal walls. After, Ghernaout et al. [11] examined the effect of the buoyancy ratio on oscillatory double-diffusive convection in binary mixture. They showed that the critical thermal Rayleigh number decreases when the solute effect is dominated, but this value increases when the thermal effect has dominated and the low value of critical thermal Rayleigh number is obtained for the case of equal opposing solute and thermal force. Recently, Ghernaout et al. [12] examined the effect of external MF on the TSNC in binary mixture for the steady and the oscillatory state. The obtained results showed a strong dependence between the structure of thermal and solutal flow and the buoyancy ratio.

To the best of our knowledge, the effect of MF on the onset of thermosolutal convection has never been examined in the literature. For this, in the present work, our interest was focused on the two transitions of the TSNC under the effect of an external MF in binary mixture: the onset of thermosolutal convection and the transition from laminaroscillatory thermosolutal flow. An external MF was applied in two directions, vertical and horizontal, separately for different Hartmann number $(H a \leq 100)$. This investigation is organized as follows: Section 2 presents the problem statement and modelling. Section 3 discusses the numerical methods and techniques which have been used for the computation. Section 4 presents the results and their discussion where the onset of two transition point of thermosolutal natural convection is determined and discussed, for different values of buoyancy ratio $(N: 0.75,1.0$, and 1.25) and different intensity-orientation of MF.

\section{PROBLEM STATEMENT AND MODELING}

The physical problem under consideration (Figure 1), is a square enclosure of $L$ length containing a binary fluid characterized by Prandtl number $(\mathrm{Pr}=0.71)$, Schmidt number $(S c=3.5)$. Different concentrations and temperatures are specified between the left $\left(C_{l}, T_{l}\right)$ and the right vertical walls $\left(C_{2}, T_{2}\right)$, where $C_{1}>C_{2}$ and $T_{1}>T_{2}$, and zero mass and heat fluxes are imposed on the remaining walls with no slip boundary conditions for all velocity components. The flow is supposed to be laminar and the binary fluid is supposed to be incompressible and Newtonian. Also, the Dufour and Soret effects are assumed to be negligible and the Boussinesq approximation is assumed to be valid. The thermo-physical properties of the fluid are estimated at a reference temperature $T_{0}$ and reference solute mass fraction $C_{0}$, which are set to be equal to $T_{2}$ and $C_{2}$, respectively.

Using the following dimensionless variables: $X=x / L$, $Y=y / L, \tau=t /\left(L^{2} / v\right), \quad U=u / L v, P=p L^{2} / \rho v^{2}$, $\theta=\left(T-T_{2}\right) /\left(T_{1}-T_{2}\right) \quad, \quad \Phi=\left(C-C_{2}\right) /\left(C_{1}-C_{2}\right) \quad, \quad$ the equations governing the conservation of mass, momentum, energy and species concentration in dimensionless form can be written as:

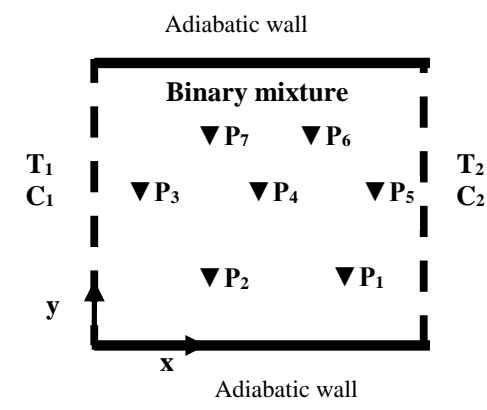

$\mathrm{B}_{y}$

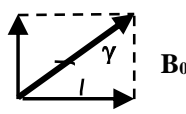

Magnetic field

(Uniform and external)

$\mathrm{B}_{\mathrm{x}}$

Figure 1. Geometry of the physical problem $\left(\mathrm{P}_{1}-\mathrm{P}_{7}\right.$ are the monitoring points)

$\nabla \cdot U=0$

$\frac{\partial U}{\partial \tau}+(U . \nabla) U=\nabla^{2} U-\nabla P+\frac{R a_{T}}{\operatorname{Pr}}(\theta-N \phi) j+F$

$\frac{\partial \theta}{\partial \tau}+U . \nabla \theta=\frac{1}{\operatorname{Pr}} \nabla^{2} \theta$

$\frac{\partial \Phi}{\partial \tau}+U . \nabla \Phi=\frac{1}{L e \times \operatorname{Pr}} \nabla^{2} \Phi$

where $j$ is the unit vector in y-direction and $F$ Lorentz force. Their components in $x$ - and $y$-directions are given the work of Bouabdallah and Bessaih [13], $U$ velocity vectors. $\theta$, dimensionless temperature, and $\Phi$ dimensionless concentration. $R a_{T}=\left(g \beta_{T} \Delta T L^{3}\right) / v \alpha$, is the thermal Rayleigh number, $N=R a_{S} / R a_{T} L e$ is the ratio of the buoyancy forces, $R a_{S}=\left(g \beta_{S} \Delta C L^{3}\right) / v \alpha$, is the solutal Rayleigh number, $\operatorname{Pr}=v / \alpha$ is the Prandtl number, $L e=\alpha / D$ is the Lewis number, $\alpha$ and $D$ are the thermal and molecular diffusivities, respectively. The initial and boundary conditions are given as:

For $t=0 ; U=V=\Theta=\Phi=0$

For $t \succ 0$;

At $\mathrm{X}=0$ and $\mathrm{X}=1,0 \leq Y \leq 1: \quad \mathrm{U}=\mathrm{V}=0$

At $\mathrm{Y}=0$ and $\mathrm{Y}=1,0 \leq X \leq 1: \mathrm{U}=\mathrm{V}=0, \frac{\partial \theta}{\partial Y}=\frac{\partial \Phi}{\partial Y}=0(5 \mathrm{c})$

At $\mathrm{X}=0,0 \leq Y \leq 1: \quad \theta=\Phi=0$

At $\mathrm{X}=1,0 \leq Y \leq 1: \quad \theta=\Phi=1$ 
The average heat and mass fluxes at the left wall are given in the dimensionless terms by Nusselt and Sherwood numbers (Ghernaout et al. [11]).

\section{NUMERICAL SOLUTION TECHNIQUE}

Equations (1-4) coupled to the boundary and initials conditions (Eqs.(5a-e)) are solved numerically using a finite volume method. The SIMPLER Algorithm was utilized to solve the velocity-pressure coupling. A second-order accurate central differencing scheme was used for approximation of convective and diffusive flux. The TDMA Algorithm was used to solve the algebraic equations system. A fully implicit time marching was realized with a fixed dimensionless time step $\left(\Delta \tau=5 \times 10^{-6}\right)$. At this stage, the steady-state solution was reached. To check and estimate the grid independence study, it will be adopted the grid $82 \times 82$, for $\varepsilon=1.07$ (where $\varepsilon$ represents the coefficient of geometric progression). For more details, it may be found in the work of (Ghernaout et al. [12]).

The computational code of the present numerical study is validated by some numerical and experimental results. A good agreement was obtained with the results of Beghein et al. [14] and Sezai and Mohamed [7]. Also, a good result was obtained in the work of Ghernaout et al. [12] with validation of the experimental results of Han and Kuehn [3-4].

\section{RESULTS AND DISCUSSIONS}

In this section, we present the numerical results of the onset of oscillatory TSNC in presence of external magnetic square enclosure. We varied the Hartmann number $\mathrm{Ha}$ from 0 to 100 (the case of $H a=0$ corresponds to the case without $\mathrm{MF})$. Lower than these conditions $(\mathrm{Ha}<100)$, the enhancement of heat transfer caused by the application of a low MF is negligible, as confirmed experimentally in liquid metal by Tagawa and Ozoe [15] and Atia et al. [16], for Hartmann number up to 100-200. Different measurement points in calculations $\left(P_{1}, P_{2}, P_{3}, P_{4} P_{5}, P_{6}\right.$ and $\left.P_{7}\right)$ were shown schematically in Figure 1. These points are located in the main areas of the cavity: the heart, near vertical walls and near horizontal walls. This finding schow a good control and measurement of flow parameters over time. All results presented herein are given in dimensionless form.

\subsection{Onset of thermosolutal natural convection}

The onset of TSNC of a binary mixture for different values of buoyancy ratio is reduced to determine the thermal Rayleigh number $R a_{T}$ that corresponds to the onset of the mass convection; this is obtained for values of Sherwood number $S h>1.1$. The onset of the thermal convection is obtained for the values of Nusselt number $N u>1.1$. The results obtained are illustrated in Figure 2. It is noted that the mass convection triggers before thermal convection whatever buoyancy ratio $(0<N<2)$, except for the case of equilibrium thermally induced forces and those of solutal origin $(N=1)$ where both mechanisms are activated in parallel $\left(R a_{T}=\right.$ 8400). At first, the fluid particles move without transport heat at low thermal Rayleigh numbers (heat transfer regime conductive). By increasing the ratio of buoyancy forces $N$, the thermal Rayleigh number $\left(R a_{T}\right)$ is in a linear growth for the values of $N<1$. In addition, for $N>1, R a_{T}$ is decreasing but with higher values. This will result in a delay of the rise of convection thermosolutal in proportion to higher values when the solutales forces are dominant compared to the case of the dominance of the thermal forces.

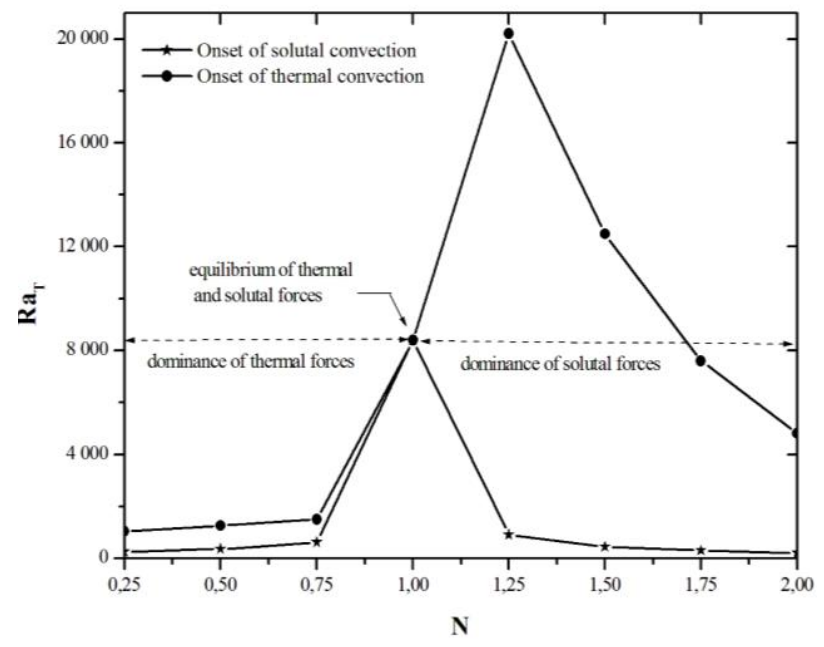

Figure 2. Onset of TSNC for different values of $N$ $(\operatorname{Pr}=0.71, S c=3.5)$.

The onset of the thermosolutal convection is presented for three values of $N$ : the first concerns the onset of mass convection manifested by changing the shape of isoconcentrations (the curvature of the iso-concentration lines is activated).

In purely conductive regime, iso-concentration lines are parallel to the vertical walls. In the second interval, this onset of the thermal convection in which the change of the shape of the isotherms occurs. This is visible for $N u$ and $S h=1.1$ (dominance of thermal forces, $N=1.75$ ).

In the last interval, the change of the isotherms and isoconcentrations begins in parallel for the case $N=1$ (cases of equilibrium thermal forces and those of solutales), and the thermosolutal convection is triggered (Figure 3). It can be see that the thermal and solutale stratification develop in a similar manner before the onset of TSNC for all the range of buoyancy ratio $(0<N<2)$. So, it can be concluded here that the mass transfer is triggered by the heat convection and the fluid particles move without transporting heat.

\subsection{Onset of thermosolutal convection under the effect of MF}

To study the influence of the external MF in onset of thermosolutal convection of a binary mixture $(\mathrm{Pr}=0.71, \mathrm{Sc}$ = 3.5), for different Hartmann number $(\mathrm{Ha}=0,10,20,30$, 40 and 50) in horizontal $\left(\gamma=0^{\circ}\right)$ and vertical directions $(\gamma=$ $90^{\circ}$ ) for $N=0.75$, it is significant to determine the thermal Rayleigh number that coincides with the onset of the mass convection $(S h>1.1)$ and the thermal Rayleigh number that corresponds to the onset of the thermal convection $(N u>1.1)$ (Figure 4).

It can be seen that the onset of TSNC is affected by the MF (Hartman numbers), producing an increase of the thermal Rayleigh number. We find also that the thermal Rayleigh that coincides to the onset of TSNC with MF applied in the horizontal direction is more important than in the case of vertical direction. As a conclusion, it can be seen that, in the onset of TSNC, the MF applied in the horizontal direction 
damped the flow better than the MF applied in the vertical direction.

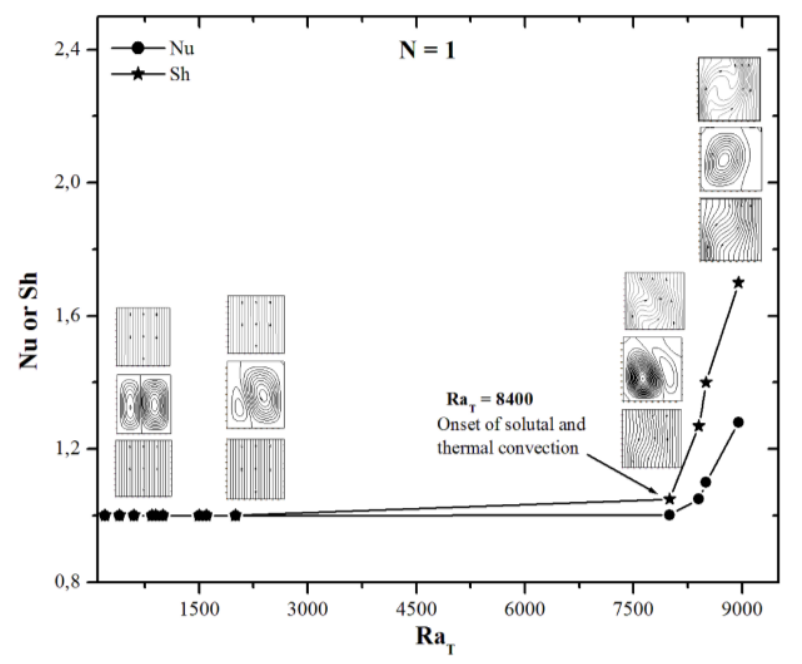

(a) $\quad N=1$

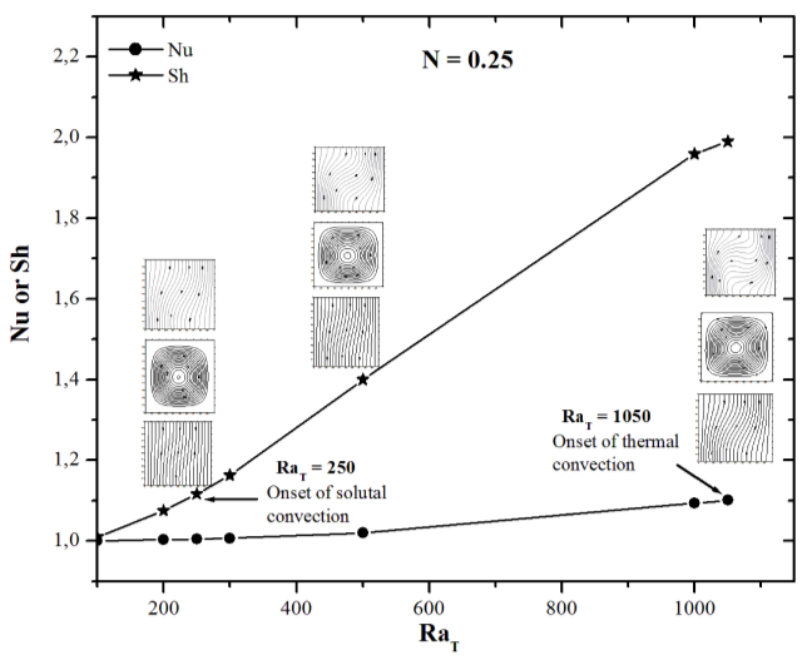

(b) $N=\mathbf{0 . 2 5}$

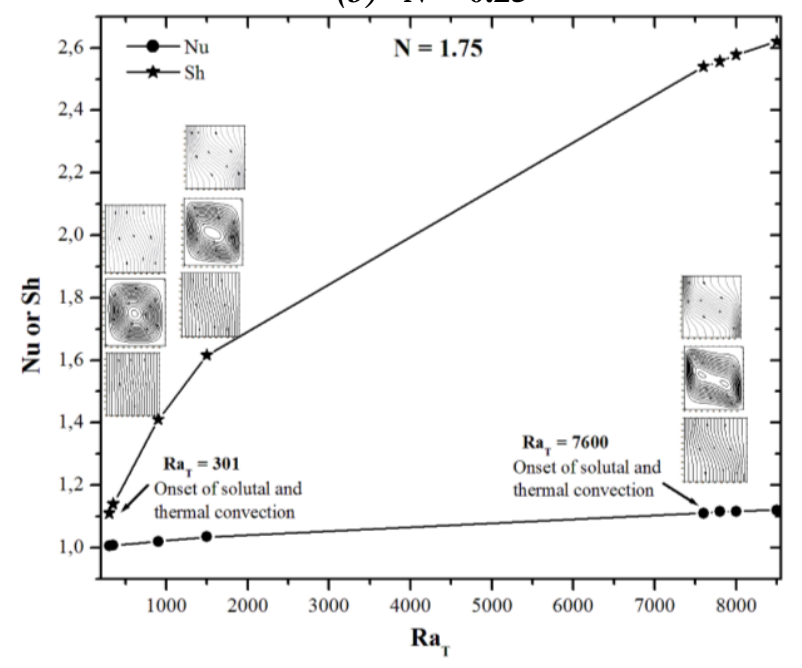

(c) $N=\mathbf{1 . 7 5}$

Figure 3. Onset of TSNC for three cases: $N=0.25,1$ and 1.75 with iso-concentrations, streamlines and isotherms at various $R a_{T}$.

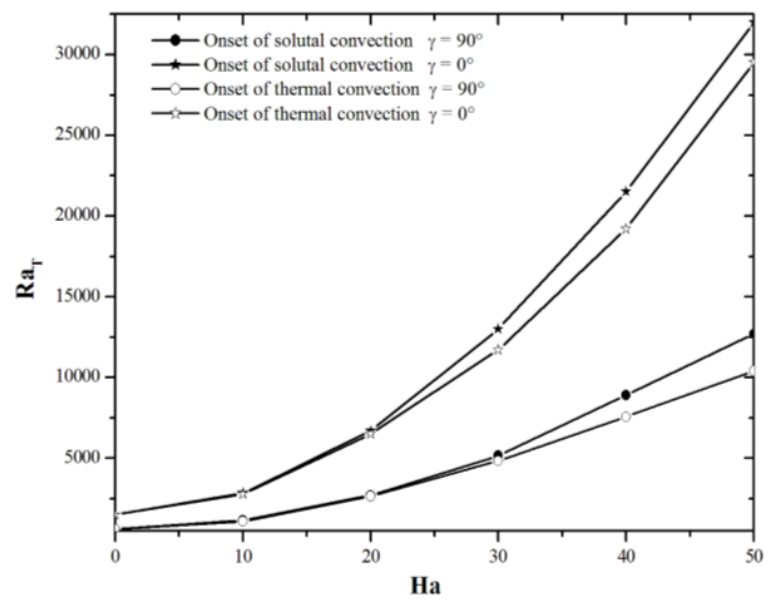

Figure 4. Onset of TSNC for different values of $\mathrm{Ha}$ in vertical and horizontal MF for $N=0.75$.

\subsection{Oscillatory flow under the effect of MF}

It is convenient to treat in this research a MF applied in two directions: horizontal $\left(B_{x}\right)$ and vertical $\left(B_{y}\right)$, and the Hartmann number takes the values $H a=0,5,10,20,30,50$ and 100. The application of the MF causes the flow damping. The increasing number of Hartmann causes amplification in the number $R a_{T C r}$. This results in the fact that the oscillatory flow regime will be behindhand by the MF so that the MF and the value of the number of $R a_{T C r}$ will be enhanced. The streamlines reflect the change of the hydrodynamic, heat and mass parameters between them. Then, for a periodic and oscillatory regime, at a point (measuring probe) considered, these changes are closed circles and reflect the periodicity of the flow regime. Furthermore, for no periodic oscillations, these changes arise to become the endpoints for the steady state or other unordered structure for unsteady state.

In Figure 5, the streamlines of temperature- concentration, for a flow double diffusion in the probe $P_{3}$, are presented. The portraits are presented for $N=1.25$ for three values of the number of thermal Rayleigh: lower value in comparison of the critical value $R a_{T}<R a_{T C r}$, and a critical value $R a_{T C r}=$ $2.1 \times 10^{5}$, and the third value greater than the critical value $R a_{T}>R a_{T C r}$. Closed circles show that the periodic system is well established for $R a_{T}=R a_{T C r}$, reflecting the periodicity of the flow regime [17]. Against by, for values of the number of upper or lower critical Rayleigh number of thermal Rayleigh, the curves do not form closed circles totally same or unordered to values lower forms. This technique has been used successively in the work of Bouabdallah et al. [10] and Ghernaout et al. [11] to confirm the established oscillatory convective flow. So as to comprehend the oscillatory manner of the different flow parameters, during a period of time, the variations of the vertical velocity component $V$ for the point $P_{2}$ are presented (Figure 6) at oscillatory thermosolutal convection regime $\left(R a_{T C r}=1.605 \times 10^{5}, \mathrm{Ha}=25, \gamma=90^{\circ}\right.$ and $N=0.75)$.

To examine the solutal and thermal flow patterns, the isotherms and iso-concentrations for a selected time in a period time: $\tau_{a}, \tau_{b}, \tau_{c}, \tau_{d}, \tau_{e}$ and $\tau_{f}$ are depicted. 


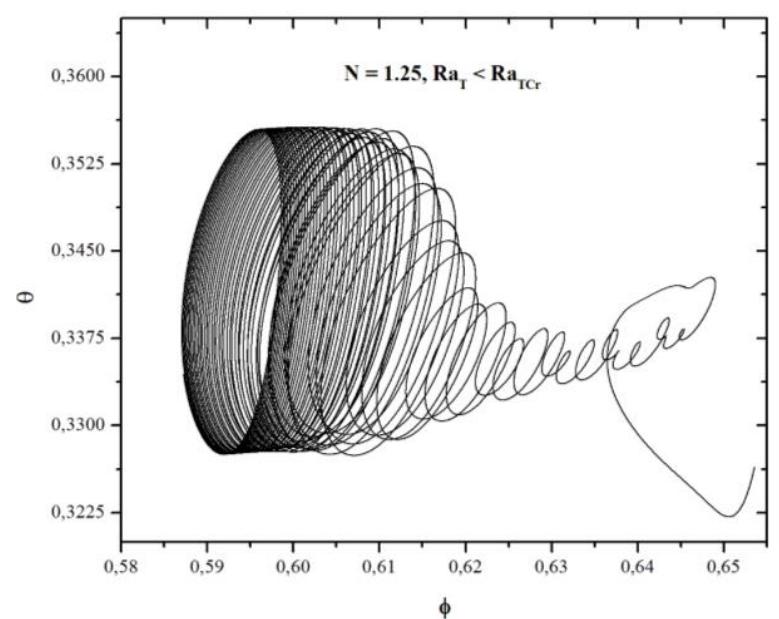

(a) $R a_{T}<R a_{T C r}$

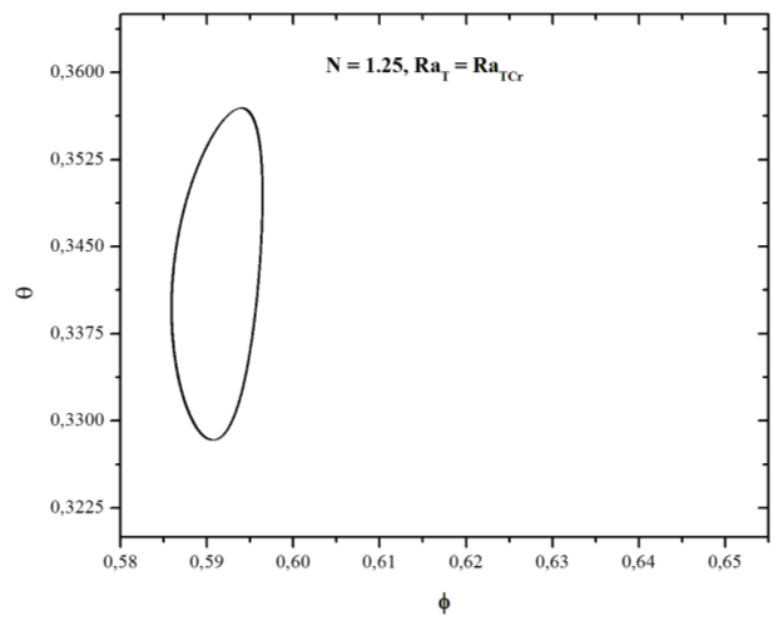

(b) $\boldsymbol{R} \boldsymbol{a}_{T}=\boldsymbol{R} \boldsymbol{a}_{T C r}$

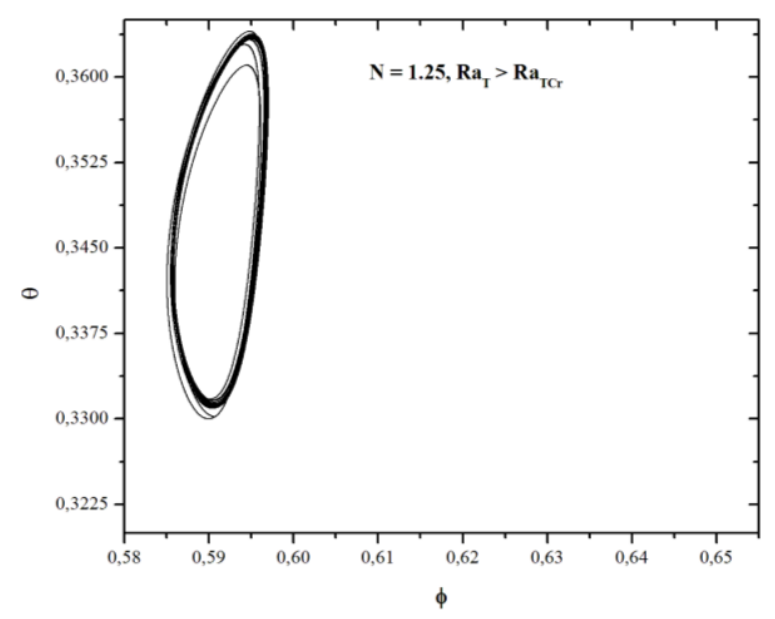

(c) $\boldsymbol{R} a_{T}>\boldsymbol{R a} a_{T C r}$

Figure 5. Streamlines of temperature - concentration for $N=$ 1.25 and $R a_{T}<R a_{T C r}$ (a), $R a_{T C r}=2.1 \times 10^{5}$ (b), $R a_{T}>R a_{T C r}$ (c).

The iso-concentrations have two small cellular at the midium of the cavity. Evidently, the structures of the flow in moments $\tau_{a}$ and $\tau_{f}$ are identical. This fact indicates that the structure of the flow is repeated in every period time. For the temporal evolution, for a period time of vertical velocity component, it notes that the iso-concentrations are closed nigh solutales gradients due to vertical walls, with the birth of two small cells in the center of the cavity and wheeled in clockwise direction with symmetric structure in each period time due to the dominance of thermal buoyancy. So, it can be distinctly seen that the structure of the isotherms is confined near the thermal gradients due to vertical walls.

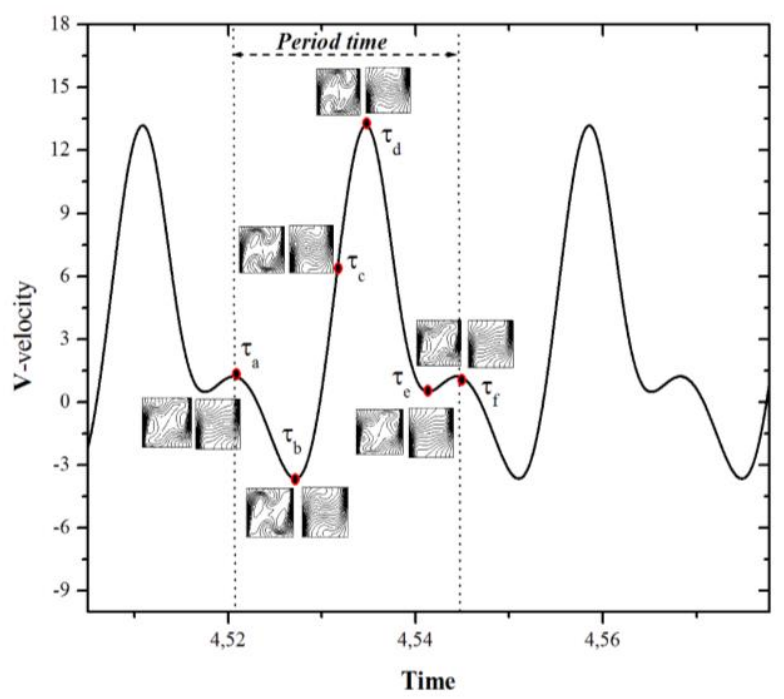

Figure 6. Time-dependent oscillatory thermosolutal flow convection in a period time with iso-concentrations and isotherms at various dimensionless time: $\tau_{a}, \tau_{b}, \tau_{c}, \tau_{d}, \tau_{e}$ and $\tau_{f}$, for $R a_{T C r}=1.605 \times 10^{5}$ and $N=0.75, H a=25$, and $\gamma=90^{\circ}$.

\section{CONCLUSION}

A TSNC of binary mixture under the effect of external MF was studied. Opposing buoyancy forces due to horizontal concentration and thermal gradients were applied. The MF was applied in two directions, vertical and horizontal, separately for different Hartmann number $(\mathrm{Ha} \leq 100)$. The finite volume method has been used to numerically solve the transport equations. The obtained results have been compared with the available data (experimental/numerical) from the literature and good agreement has been found. The main results obtained in this study are as follows:

- The onset of TSNC is determined for different buoyancy ratios, according to the dominating of thermalequilibrium-solutale force.

- The mass convection triggers before thermal convection whatever buoyancy ratio $(0<N<2)$, except for the case of equilibrium thermally induced forces and those of solutal origin $(N=1)$ where both mechanisms are activated in parallel.

- The increasing of Hartmann number causes an increase in the number $R a_{T C r}$, which results in the fact that the oscillatory flow regime will be delayed by the MF so that the MF and the value of $R a_{T C r}$ will be increased.

In the onset of TSNC, the MF applied in the horizontal direction damped the flow best than the MF applied in the vertical direction.

\section{REFERENCES}

[1] Lee J., Hyen M.T., Kim K.W. (1988). Natural convection in confined fluids with combined horizontal temperature and concentration gradients, 
Int J Heat Mass Transf, Vol. 31, No. 10, pp. 19691977. DOI: $\underline{10.1016 / 0017-9310(88) 90106-8}$

[2] Kamotani Y., Wang J.S., Ostrach S., Jiang H.D. (1985). Experimental study of natural convection in shallow enclosures with temperature and concentration gradients, Int J Heat Mass Transf, Vol. 28 , No. 1 , pp. $165-173$, DOI: $10.1016 / 0017-$ 9310(85)90018-3

[3] Han H., Kuehn H.T. (1991a). Double diffusive natural convection in a vertical rectangular enclosure - II numerical study, Int J Heat Mass Transf, Vol. 34, No. 2, pp. 461-471. DOI: 10.1016/0017-9310(91)90265-G

[4] Han H., Kuehn H.T. (1991b). Double diffusive natural convection in a vertical rectangular enclosure- I experimental study, Int J Heat Mass Transf, Vol. 34, No. 2, pp. 449-459. DOI: $\underline{10.1016 / 0017-}$ 9310(91)90264-F

[5] Chen Z.W., Li Y.S., Zhan J.M. (2012). Onset of oscillatory double-diffusive buoyancy instability in an inclined rectangular cavity, Int J Heat Mass Transf, Vol. 55, pp. 3633-3640. DOI 10.1016/j.ijheatmasstransfer.2012.02.056

[6] Zhou H., Zebib A. (1994). Oscillatory doublediffusive convection in crystal growth, J Cryst Growth, Vol. 135, pp. 587-593. DOI: 10.1016/0022$\underline{0248(94) 90151-1}$

[7] Sezai I., Mohamad A.A. (2000). Double diffusive convection in a cubic enclosure with opposing temperature and concentration gradients, Phys Fluids, Vol. 12, No. 9, pp. 2210-2223. DOI: $\underline{10.1063 / 1.1286422}$

[8] Maatki C., Kolsi L., FOztop H., Chamkha A., Borjini M.N., Aissia H.B., Al-Salem K. (2013). Effects of magnetic field on 3D double diffusive convection in a cubic cavity filled with a binary mixture, Int Commun Heat Mass, Vol. 49, pp. 86-95. DOI: $\underline{10.1016 / j . i c h e a t m a s s t r a n s f e r .2013 .08 .019}$

[9] Ghachem K., Kolsi L., Mâalki C., Hussein A.K., Borjini M.N. (2012). Numerical simulation of threedimensional double diffusive free convection flow and irreversibility studies in a solar distiller, Int Commun
Heat Mass, Vol. 39, pp. 869-876. DOI: 10.1016/j.icheatmasstransfer.2012.04.010

[10] Bouabdallah S., Bessaih R. (2012). Effect of magnetic field on 3D flow and heat transfer during solidification from a melt, Int J Numer Method H, Vol. 37, pp. 154166. DOI: 10.1016/j.ijheatfluidflow.2012.07.002

[11] Ghernaout B., Bouabdallah S., Benchatti A., Bessaih R. (2014). Effect of the buoyancy ratio on oscillatory double-diffusive convection in binary mixture, Numer Heat $\operatorname{Tr} A$-Appl, Vol. 66 No. 8, pp. 928-946. DOI: 10.1080/10407782.2014.892386

[12] Ghernaout B., Bouabdallah S., Teggar M., Benniche H. (2015). Double diffusive natural convection in binary mixture under the effect of external magnetic field: steady and oscillatory state, Int J Heat \& Tech, Vol. 33, No. 4, pp. 11-18. DOI: 10.18280/ijht.330402

[13] Bessaih R., Bouabdallah S. (2008). Numerical study of oscillatory natural convection during solidification of a liquid metal in a rectangular enclosure with and without magnetic field, Numer Heat Tr A-Appl, Vol. 54, pp. 331-348. DOI: 10.1080/10407780802084660

[14] Beghein C., Haghighat F., Allard F. (1992). Numerical study of double diffusive natural convection in a square cavity, Int. J. Heat Mass Transfer, Vol. 35, No. 4, pp. 833-846. DOI: $10.1016 / 0017-9310(92) 90251-\mathrm{M}$

[15] Tagawa T., Ozoe H. (1998). Enhanced heat transfer rate measured for natural convection in liquid Gallium in a cubical enclosure under a static magnetic field, $J$ Heat Trans, Vol. 120, No. 4, pp. 1027-1032. DOI: $\underline{10.1115 / 1.2825886}$

[16] Atia A., Ghernaout B., Bouabdallah S., Bessaïh R. (2016). Three-dimensional oscillatory mixed convection in a Czochralski silicon melt under the axial magnetic field, Appl Therm Eng, Vol. 105, pp. 704-715. DOI: 10.1016/j.applthermaleng.2016.03.087

[17] Liu J.P., Tao W.Q. (1999). Bifurcation to oscillatory flow of the natural convection around a vertical channel in rectangular enclosure, Int. J. Numer. Method H, Vol. 9, No. 2, pp. 170-185. DOI: $\underline{10.1108 / 09615539910256027}$ 